\title{
Mesenchymal stem cells treatment in COVID-19 patient with multi-organ involvement
}

\author{
Yilmaz R ${ }^{1}$, Adas $\mathrm{G}^{2,3}$, Cukurova $\mathrm{Z}^{1}$, Kart Yasar $\mathrm{K}^{4}$, Isiksacan $\mathrm{N}^{5}$, Oztel ON ${ }^{6}$, Karaoz E $\mathrm{E}^{6,7,8}$ \\ Department of Biochemistry and Immunology, Bakirkoy Dr. Sadi Konuk Education and Research Hospital, \\ Health Science University, Istanbul, Turkey. nisiksacan@gmail.com
}

\section{ABSTRACT}

The aim of this study is to evaluate the therapeutic effect of mesenchymal stem cells (MSCs) in a severe case of brain and multiple organ involvement in a patient with COVID-19. Here, a 51-year-old male patient with multi-organ involvement due to COVID-19 infection and developing cardiac arrest is presented. MSCs were transplanted to the patient four times systematically and once intrathecally. As a result, the application of MSCs has been found to have a healing effect on organs in this patient with severe COVID-19 infection. In addition, transplantation of MSCs both systematically and intrathecally is considered to be effective in the treatment of the central nervous system (Tab. 2, Fig. 2, Ref. 24). Text in PDF www.elis.sk KEY WORDS: mesenchymal stem cell, COVID-19, organ involvement.

\section{Introduction}

Clinical symptoms of COVID-19 infection include fever, cough, and myalgia or fatigue with pneumonia demonstrated on chest CT scan imaging (1). The clinical situation of patients varies from mild fever to acute respiratory distress syndrome (ARDS) and death $(2,3)$. Patients with severe disease were likely to display neurologic symptoms such as acute cerebrovascular diseases, impaired consciousness, loss of smell and taste, and skeletal muscle injury $(2,4)$.

Mesenchymal stem cells (MSCs) have been widely used in cell-based therapy, from basic research to clinical trials $(5,6,7)$. Wharton's jelly mesenchymal stem cells (WJ-MSCs) have distinct advantages of being abundant, easy to obtain with minimal invasiveness, and readily cultured in a sufficient number for trans-

\footnotetext{
${ }^{1}$ Department of Anesthesia and Intensive Care, Bakirkoy Dr. Sadi KonukEducation and Research Hospital, Health Science University, Istanbul, Turkey, ${ }^{2}$ Department of Surgery, Bakirkoy Dr. Sadi Konuk Education and Research Hospital, Health Science University, Istanbul, Turkey, ${ }^{3}$ Stem Cell and Gene Therapies Center, Health Science University, Istanbul, Turkey, ${ }^{4}$ Department of Infectious Diseases, Bakirkoy Dr. Sadi Konuk Education and Research Hospital, Health Science University, Istanbul, Turkey, ${ }^{5}$ Department of Biochemistry and Immunology, Bakirkoy Dr. Sadi Konuk Education and Research Hospital, Health Science University, Istanbul, Turkey, ${ }^{6}$ Liv Hospital, Center for Regenerative Medicine and Stem Cell Manufacturing (LivMedCell), İstanbul, Turkey, ${ }^{7}$ Faculty of Medicine, Department of Histology \& Embryology, Istinye University, Istanbul, Turkey, and ${ }^{8} \mathrm{Center}$ for Stem Cell and Tissue Engineering Research \& Practice, Istinye University, Istanbul, Turkey

Address for correspondence: N. Isiksacan, Department of Biochemistry and Immunology, Bakirkoy Dr. Sadi Konuk Education and Research Hospital, Zuhuratbaba Mahallesi, Tevfik Saglam Caddesi, No: 11, 34000, Istanbul, Turkey.
}

plantation without ethical issues of allografting (8). The aim of the case report is to determine WJ-MSCs treatment's efficacy in a severe case of multiple organ failure and brain involvement in a patient with COVID-19 infection. In addition, another aim of the study is to get to understand the way in which MSCs are given, especially in patients with brain involvement.

\section{Case report}

This study was undertaken in the Bakirkoy Dr. Sadi Konuk Education and Training Hospital at Health Science University and Istinye University with the permission of the Ministry of Health. The study protocol was approved by the Ethical Committee. The informed consent was obtained from the patient's relatives.

A 51-year-old male patient started to complain of cough, myalgia, high fever $\left(39.5^{\circ} \mathrm{C}\right)$, and diarrhea on March 11, 2020. Upon the progression of symptoms, the patient was hospitalized in the infection service on March 16. Thorax tomography was performed and throat swab was taken. Radiological involvement related to bilateral COVID-19 infection was observed in both lungs (Fig. 1A). The patient had no previous chronic illnesses or additional pathologies in his medical records. A swab was taken, and the diagnosis of COVID-19 was made using the PCR method. A supportive therapy was initiated. Upon progression of respiratory distress during his daily follow-up, the patient was transferred to the intensive care unit (ICU).

During the ICU follow-up, the patient was intubated oro-tracheally due to high fever; DSS: 33-40 breaths per minute (bpm), low $\mathrm{O}_{2}$ saturation, and gradually increasing respiratory distress. Due to hypoxemia in arterial blood gas (ABG) values and deterioration in $\mathrm{P} / \mathrm{F}$ (Horowitz) values $(<150)$, the patient was placed in a prone position immediately after intubation. Methylpredniso- 

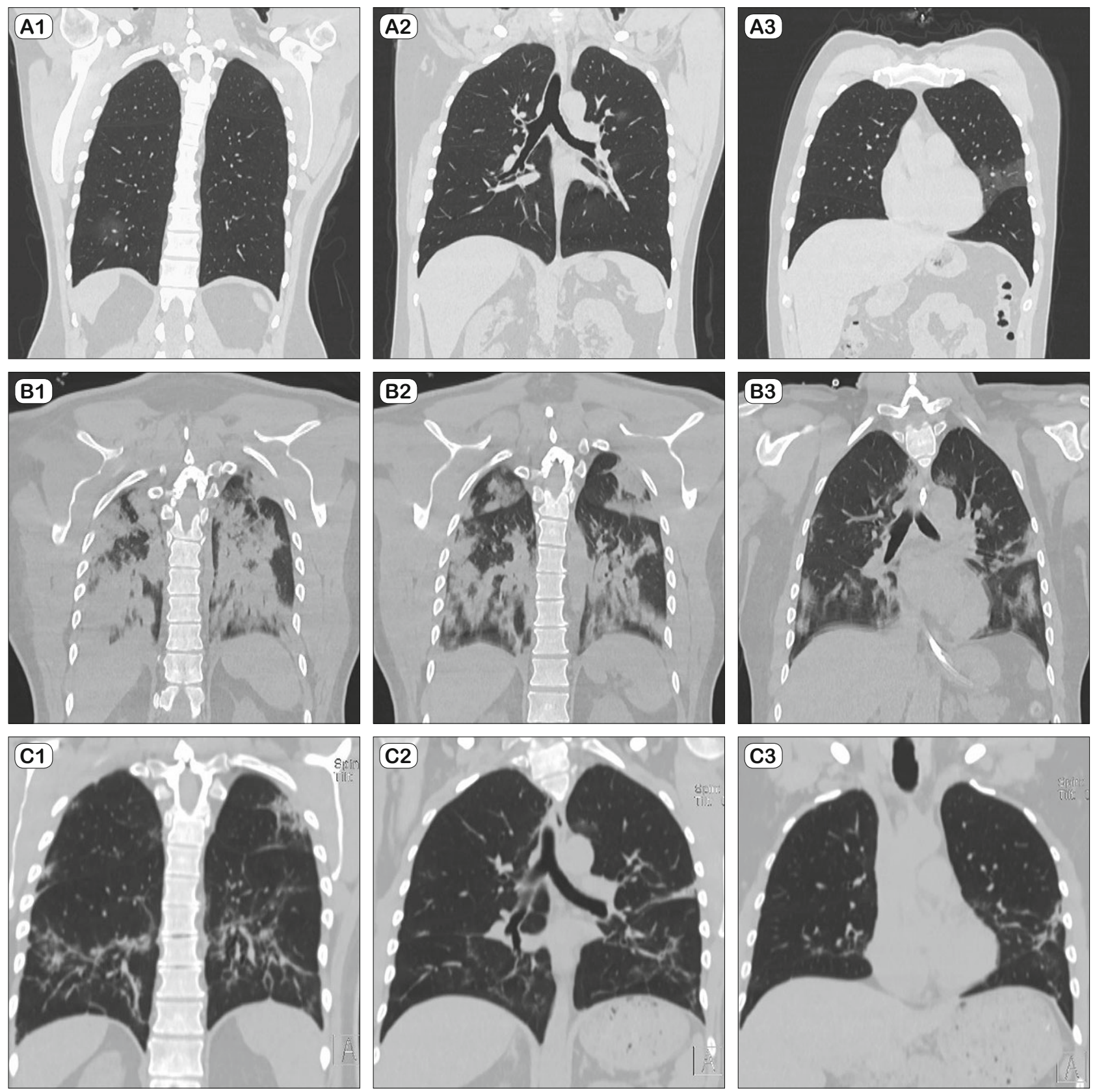

Fig. 1. Radiological evaluation of patient lungs. A1-3: focal ground-glass opacity in the lower part of the lung observed on day 5 after first symptom onset. B 1-3:on day 10 day after first symptom onset, it is observed that lesions increase in both upper and lower lung lobes. It is seen that the lesions are converted into consolidation form and merge. C 1-3: on day 25 after first symptom onset, interlobular and intralobular septal thickening is observed in parenchyma of both lungs. In his previous film, the common consolidation areas and patched ground-glass densities are observed to be resorbed.

lone was added to the treatment of the patient for 5 days. In the prone position, CPR was applied to the patient who developed sudden cardiac arrest for 10 minutes. Two hours after the arrest, a targeted temperature management was started. Body temperature was adjusted to 33 degrees. It was sedated to be between 20 and 40 according to patient state index. The antiviral treatment of the patient was changed to Avigan. The patient was thought to have a cytokine storm. On March 25, the patient was treated with tocilizumab (IL-6 antagonist) for 2 days.

Echocardiography performed after cardiac arrest revealed a global dyskinesia compatible with myocarditis, and ejection fraction (EF) of $25 \%$ with severe apical akinesia. The patient deve- 

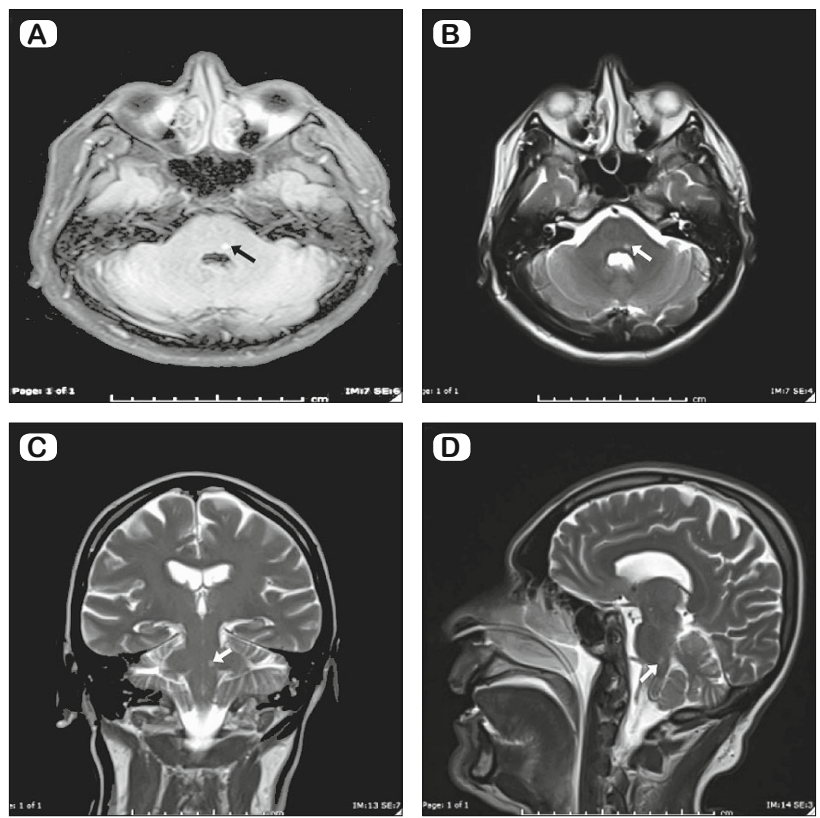

Fig. 2. Radiological evaluation of the patient brain. A-D Focal intensity increased in T2-weighted levels in the posterior side of the pons. The lesion is marked with an arrow.

loped a severe bilateral pneumonia (Fig. 1B), ARDS, and multiple organ failure, while no response was received.

The patient did not wake up and did not show any clinical improvement, even though his medical sedations were closed after the entire treatment period. On monitorization, delta waves were observed, and contrast enhanced cranial MR and CT were taken. Hyperintense signal change in T2A-FLAIR sequence in pons was observed in cranial MR (Fig. 2). After the general condition of the patient had not improved as a result of treatment, it was decided by the medical committee to perform MSC transplantation. WJMSCs were transplanted to the patient four times intravenously (IV). No side effects were observed in the patient. After stem cell transplantation, the patient underwent control echocardiography. Cardiac activity and myocardial structure were found to be normal. EF was evaluated as $60 \%$.

On April 07, 2020, the patient was extubated with high-flow nasal cannula. Upon neurological examination, the patient was observed to be orientated and cooperative. After 5 days of follow-up, the patient was taken to medical service. The patient was diagnosed with upper gastrointestinal bleeding. After the patient's anti-thromboembolic treatment had been discontinued, his vital signs were stable, and it was decided to follow him up at the clinic. The patient was discharged from hospital on April 20, 2020 as symptom-free and with no complaints. Information about the patient's laboratory examination results are given in Table 1 .

Drug treatment of the patient was made on the basis of international literature and guideline prepared by the scientific committee related to this issue and affiliated to the ministry of health. The MSCs were slowly drawn into the syringe without pressure, suspended in $250 \mathrm{ml}$ of $0.9 \% \mathrm{NaCl}$, and then given intravenously over 1 hour (Tab. 2).

The levels of alanine transaminase, aspartate transaminase, total protein, albumin, total bilirubin, direct bilirubin, ferritin, triglycerides, D-dimer, troponin I, myoglobin, procalcitonin, ammonia, c-reactive protein, pro B-type natriuretic peptide, creatine kinase, and alkaline phosphatase were determined in venous blood samples using Beckman Coulter AU5800 analyzer (Beckman Coulter, Brea, CA, USA). The complete blood count was analyzed with ADVIA 2120 i autoanalyzer (Siemens Healthcare Diagnostics, Erlangen, Germany).

For coagulation assay, all analytical procedures were carried out on a random-access coagulation analyzer (Beijing Succeeder Technology Inc. China).

Flow cytometric analyses were performed with Navios cytometer (BECLS)-Kaluza Software. Whole blood was stained with anti-human FITC-CD45, PE-CD4, ECD-CD8, PE-CY5.5-CD3 (Beckman Coulter, Brea, California) antibodies.

All samples of WJ-MSCs as cell therapy medicinal products were isolated, expanded, and analyzed in cGMP-certified facility at Liv Hospital Center for Regenerative Medicine and Stem Cell Manufacturing (LivMedCell). Human WJ-MSCs were prepared and tested as described in our previous clinical trials $(8,9,10)$.

Cryopreserved vials from each donor were thawed and mixed in the same tubes before seeded at a cell density of $4,000 \mathrm{cells} / \mathrm{cm}^{2}$. After harvest at the fourth passage, quality control tests were performed, such as flow cytometry analysis, endotoxin, rapid microbiological and sterility tests. The final product was prepared as $3 \times 10^{6}$ cells $/ \mathrm{kg} /$ dose of allogeneic WJ-MSCs pooled from three donors.

\section{Discussion}

The central nervous system (CNS) is not immune to alterations that lead to neurological disease resulting from acute, persistent or latent viral infections (11). In some circumstances, opportunistic viral pathogens such as human corona viruses can avoid the immune response and cause more severe respiratory diseases or even spread to other tissues including the CNS (12). The detection of HCoV-RNA in human brain samples clearly demonstrates that these respiratory pathogens are naturally neuroinvasive in humans, and suggests that they establish a persistent infection in human CNS (13). On March 4, 2020, researchers from Beijing Ditan Hospital, China, first described a confirmed patient with 2019-nCoV, whose cerebrospinal fluid (CSF) tested positive for 2019-nCoV by gene sequencing, suggesting a need to consider direct infection when patients with 2019-nCoV present with neurological disorders (14).

In this study, MSCs transplantation was successfully performed both systemically and intrathecally. Here, after the first 2 transplants had been given systematically, the patient was awakened and extubated. Later, the patient who developed neurological symptoms was reintubated. After that, the transplantation of MSCs was performed systematically for the third time, as well as an intrathecal stem cell transplantation. We thought the blood brain barrier (BBB) was an obstacle here. Neurologically, the dia- 


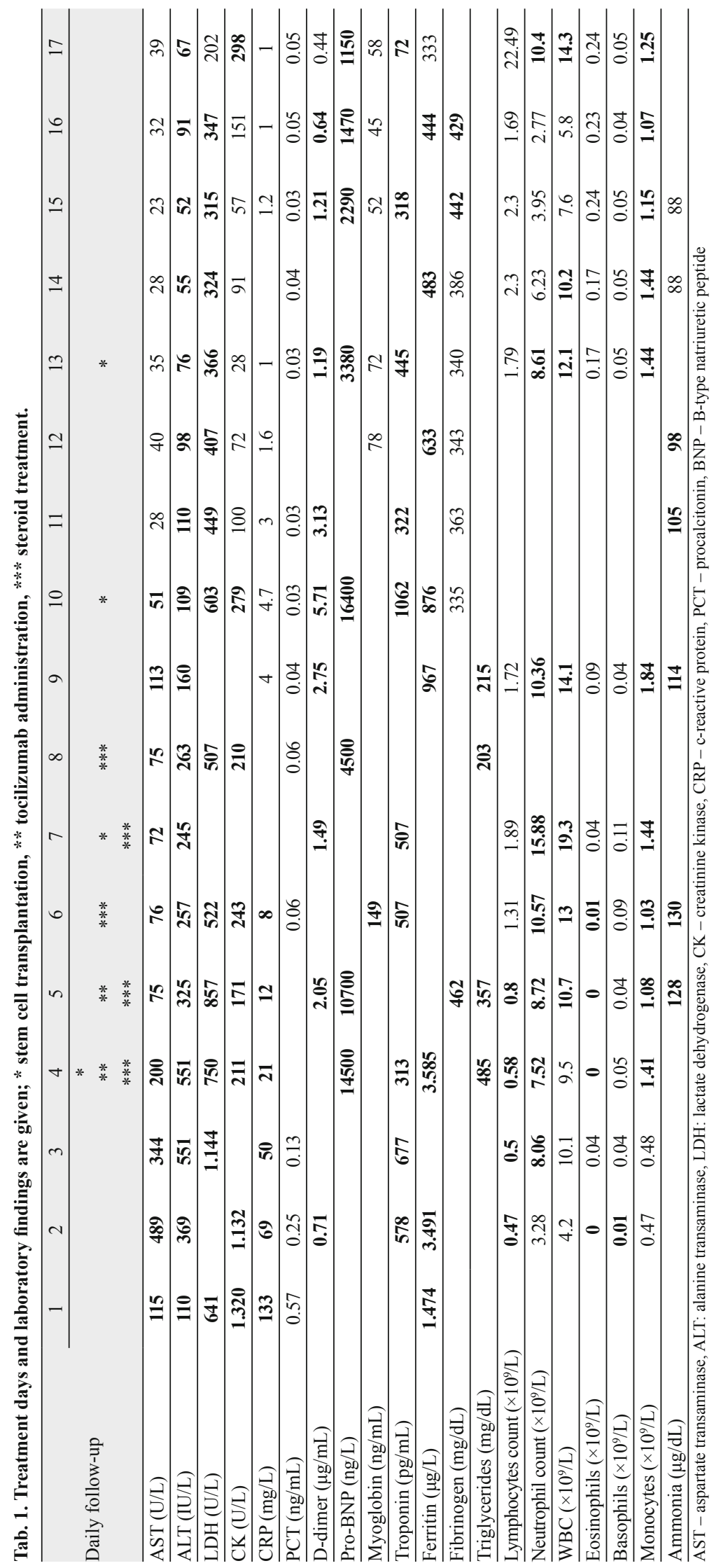

gnosis of brain involvement was demonstrated by MR and proved by COVID-19 antibodies in the spinal fluid. Upon examination of CSF samples, protein and IgG levels were elevated while albumin levels were found low. The CSF result is compatible with previous SARSCoV-2 infection studies (15). The patient's vital signs were improved, especially after intrathecal and systemic MSCs transplantations. After the patient had been extubated, his neurological symptoms regressed, consciousness restored, and he could speak. Most neurologic manifestations occurred early in the illness (the median time to hospital admission was 1-2 days) (16). In our patient, the time period between hospitalization and determining the neurological symptoms was approximately 11 days.

After transplanting MSCs through intravenous infusion, a part of the MSCs accumulate in the lung, which could potentially improve the pulmonary microenvironment, protect alveolar epithelial cells, prevent pulmonary fibrosis, and improve the lung function $(2,6,14)$. They promote tissue and organ regeneration via secreting a variety of paracrine factors, conferring anti-inflammatory, immunomodulatory, angiogenic, antifibrotic, and structural reparative properties $(17,18)$. BM-MSCs significantly improved the efficiency of cardiomyogenesis and cardiac function $(19,20)$. When the treatment of patients with organ failure due to COVID-19 infection is examined, no treatment can be seen to improve the organ damage. Our patient had cardiac involvement due to COVID-19 infection. In our patient who underwent echocardiography after cardiac arrest, the ejection fraction was very low $(25 \%)$. In echocardiography performed after systematic transplantation of MSCs, this rate increased to the level of $60 \%$. It is our opinion that the transplantation of MSCs has a healing effect on the heart. After MSCs transplantation had been performed for the fourth time, the patient's heart functions have returned to normal. Here, we think that MSCs have a positive and accelerating effect upon other healing mechanisms of the body.

MSCs engraftment was observed in the injured lung and engraftment rates increased with the extent of tissue injury (6). In a study by Leng $\mathrm{Z}$ et al, 7 patients with COVID-19 infections were given MSCs systematically, which had a healing effect (20). When the thorax CTs of the patient were examined in our case study, it was observed that the upper and lower lobes 
Tab. 2. Drug and mesenchymal stem cell management.

\begin{tabular}{|c|c|c|c|}
\hline & & & Route of administration \\
\hline \multirow[t]{7}{*}{ Drug } & Ritonavir + Lopinavir & $2 \mathrm{X} 400 \mathrm{mg}$ & \multirow{5}{*}{ Oral } \\
\hline & Oseltamivir & $2 \times 75 \mathrm{mg}$ & \\
\hline & Azithromycin & $1 \mathrm{X} 500 \mathrm{mg}$ & \\
\hline & Favipiravir & $2 \times 1600 \mathrm{mg} / 2 X 600 \mathrm{mg}$ & \\
\hline & Hydroxychloroquine & $2 \times 200 \mathrm{mg} /$ & \\
\hline & Methylprednisolone & $2 \mathrm{X} 100 \mathrm{mg}$ & \multirow{2}{*}{ Intravenous } \\
\hline & Tocilizumab & $1 \mathrm{X} 400 \mathrm{mg}$ & \\
\hline \multirow[t]{4}{*}{$\overline{\mathrm{MSC}}$} & 1st application/ Day 1 & $3 \times 10^{6} / \mathrm{kg}$ & \multirow{3}{*}{ Intravenous } \\
\hline & 2nd application/ Day 3 & $3 \times 10^{6} / \mathrm{kg}$ & \\
\hline & 3rd application/ Day 6 & $3 \times 10^{6} / \mathrm{kg}$ & \\
\hline & 4th application/ Day 9 & $2 \times 10^{6} / \mathrm{kg}+1 \times 10^{6} / \mathrm{kg}$ & Intravenous + intrathecal \\
\hline
\end{tabular}

due to COVID-19 infection will increase the effectiveness of treatment.

\section{References}

1. Kruse LR. Therapeutic strategies in an outbreak scenario to treat the novel coronavirus originating in Wuhan, China (version 2; peer review: 2 approved) Research 2020.

2. Sun T, Guan J. Novel coronavirus and central nervous system. Eur J Neurol 2020. DOI: 10.1111/ene.14227.

3. Ozturk S, Elcin EA, Elcin MY. Mesenchymal stem cells for coronavirus (COVID-19) induced pneumonia: Revisiting the paracrine hypothesis with new hopes. Aging Dis 2020. DOI: 10.14336/ AD.2020.0403. of both lungs were commonly held, especially in the second thorax CT. In our patient, the lesions seen in both lungs had groundglass appearance, and areas of consolidation were compatible with COVID-19 infection $(21,22)$. After the MSC transplantation for the last time in our patient, bilateral lung symptoms regressed on control thorax CT. After the patient had been discharged, there was no problem in his medical checkups.

After the first MSC transplantation in our patient, the values of AST, ALT, LDH, CK, pro-bnp, ferritin, triglyceride, fibrinogen, ammonia, and myoglobin began to decrease. The second time the MSCs had been given, CRP reached normal values (Tab. 1). We thought that MSCs were related to the immunomodulatory effect on cytokine storm. On day 2 after MSC transplantation, the lymphocyte count reached the normal level. What is important here, is that reaching the efficacy of lymphocyte count on day 2 was achieved by the administration of MSCs and tocilizumab at the same time. In the literature, it is seen that the normal level of the number of lymphocytes was reached on day 5 in the study of Xiaoling Xu et al (23). In our study, the increase in lymphocytes was thought to be related to MSCs transplantation. It was observed that the number of TH-2 cells increased, and the number of TH-1 cells decreased in the immune modulation after MSC transplantation. After the first MSC transplant, the proportions of CD4+T cells and CD8+T cells were $66 \%$ and $26.7 \%$, respectively. After $3 \mathrm{MSC}$ transplantation, the proportion of CD4+T cells was $42.9 \%$ and $39.1 \%$, while that of CD8+T cells was $18.7 \%$ and $22 \%$, respectively. After the second and third MSC transplantations, it was found that the proportion of CD4+T cells was $42.9 \%$ and $39.1 \%$ while that of CD $8+$ cells was $18.7 \%$ and $22 \%$, respectively. In previous studies, the decrease in $\mathrm{T}$ lymphocytes has been shown to be due to co-death in T cells infected with the virus $(6,24)$.

\section{Learning points}

The transplantations of MSCs, both systematically and intrathecally, were effective in the treatment of the central nervous system. This activity is related to the fact that MSCs administered intrathecally can easily cross the blood brain barrier. Combining both ways of MSC treatment in multi-organ and brain involvement
4. Li YC, Bai WZ, Hashikawa T. Response to Commentary on "The neuroinvasive potential of SARS-CoV-2 may play a role in the respiratory failure of COVID-19 patients". J Med Virol 2020. DOI: 10.1002/jmv.25824.

5. Atluri S, Manchikanti L, Hirsch AJ. Expanded umblical cord mesenchymal stem cells (UC-MSCs) as a therapeutic strategy in managing critically ill COVID-19 patients: the case for compassionate use. Pain Physician 2020: 71-83.

6. Behnke J, Kremer S, Shahzad T et al. MSC based therapies-new perspectives for the injured lung. J Clin Med 2020; 9 (3): 682. https://DOI. org/10.3390/jcm9030682.

7. Kabatas S, Demir CS, Civelek E, Yilmaz I, Kircelli A, Yilmaz C, Akyuva Y, Karaoz E. Neuronal regeneration in injured rat spinal cord after human dental pulp derived neural crest stem cell transplantation. Bratisl Lek Listy 2018; 119 (3): 143-151. DOI: 10.4149/BLL_2018_028.

8. Kabataş S, Civelek E, Inci C, Yalçınkaya EY, Gunel G, Kır G, Albayrak E, Ozturk E, Adas G, Karaoz E. Wharton's Jelly-Derived Mesenchymal Stem Cell Transplantation in a Patient with Hypoxic-Ischemic Encephalopathy: A Pilot Study. Cell Transplant 2018; 27 (10): 1425-1433. DOI: $10.1177 / 0963689718786692$.

9. Dai A, Baspinar O, Yeşilyurt A, Sun E, Aydemir Çİ, Öztel ON, Capkan DU, Pinarli F, Agar A, Karaöz E. Efficacy of Stem Cell Therapy in Ambulatory and Nonambulatory Children with Duchenne Muscular Dystrophy- Phase I-II. Degenerative Neurol Neuromusc Dis 2018; 8: 63-77. eCollection 2018. https://www.ncbi.nlm.nih.gov/pubmed/30498389.

10. Okur SÇ, Erdoğan S, Demir CS, Günel G, Karaöz E. The Effect of Umbilical Cord-Derived Mesenchymal Stem Cell Transplantation in a Patient with Cerebral Palsy: A Case Report. Internat J Stem Cells 2018; 11 (1): 141-147. https://www.ncbi.nlm.nih.gov/pubmed/29699386.

11. Desforges M, Le Coupanec A, Dubeau P, Bourgouin A, Lajoie L, Dubé M, Talbot PJ. Human Coronaviruses and Other Respiratory Viruses: Underestimated Opportunistic Pathogens of the Central Nervous System? Viruses 2019; 12 (1). pii: E14. DOI: 10.3390/v12010014.

12. Desforges M, Le Coupanec A, Stodola JK, Meessen-Pinard M, Talbot PJ. Human coronaviruses: viral and cellular factors involved in neuroinvasiveness and neuropathogenesis. Virus Res 2014; 194: 145-158.

13. Desforges M, Le Coupanec A, Brison E, Meessen-Pinard M, Talbot PJ. Neuroinvasive and neurotropic human respiratory coronaviruses: potential neurovirulent agents in humans. Adv Exp Med Biol 2014; 807: 75-96. DOI: 10.1007/978-81-322-1777-0_6. 


\section{7-852}

14. Sun T, Guan J. Novel coronavirus and central nervous system. Eur J Neurol 2020. DOI: 10.1111/ene.14227.

15. Helms J, Kremer S, Merdji H, Clere-Jehl R, Schenck M, Kummerlen $\mathbf{C}$ et al. Neurologic Features in Severe SARS-CoV-2 Infection. New Engl J Med 2020. DOI: 10.1056/NEJMc2008597.

16. Mao L, Jin H, Wang M. Neurologic manifestations of hospitalized patients with coronavirus disease 2019 in Wuhan, China. JAMA Neurol. 2020

17. Han J, Li Y, Li Y. Strategies to enhance Mesenchymal Stem CellBased therapies for acute respiratory distress syndrome. Stem Cells International 2019: 1-12.

18. Adas G, Koc B, Adas M, Duruksu G, Subasi C, Kemik O et al. Effects of mesenchymal stem cells and VEGF on liver regeneration following major resection. Langenbecks Arch Surg 2016; 401 (5): 725-740. DOI: $10.1007 / \mathrm{s} 00423-016-1380-9$.

19. Shinmura D1, Togashi I, Miyoshi S, Nishiyama N, Hida N, Tsuji H et al. Pretreatment of human mesenchymal stem cells with pioglitazone improved efficiency of cardiomyogenic transdifferentiation and cardiac function. Stem Cells 2011; 29 (2): 357-366. DOI: 10.1002/stem.574.
20. Gojo S, Gojo N, Takeda $\mathrm{Y}$ et al. In vivo cardiovasculogenesis by direct injection of isolated adult mesenchymal stem cells. Exp Cell Res 2003; 288: 51-59.

21. Shi H, Han X, Jiang $\mathbf{N}$ et al. Radiological findings from 81 patients with COVID-19 pneumonia in Wuhan, China: a descriptive study. Lancet Infect Dis 2020.

22. Salehi S, Abedi A, Balakrishnan S, Gholamrezanezhad A. Coronavirus Disease 2019 (COVID-19): A Systematic Review of Imaging Findings in 919 Patients. AJR: 215, July 2020.

23. Xiaoling $\mathbf{X}$, Mingfeng $\mathbf{H}$, Tiantian $\mathbf{L}$ et al. Effective Treatment of Severe COVID-19 Patients with Tocilizumab. chinaXiv:202003.00026v1.

24. Wang X, Xu W, Hu G, Xia S, Sun Z, Liu Z et al. SARS-CoV-2 infects $\mathrm{T}$ lymphocytes through its spike protein-mediated membrane fusion. Cell Mol Immunol 2020. DOI: 10.1038/s41423-020-0424-9.

Received May 19, 2020. Accepted May 27, 2020. 